\title{
A novel mutation in the RS1 gene in a Chinese family with $\mathrm{X}$-linked congenital retinoschisis
}

\author{
NA ZHANG ${ }^{*}$, YAO PENG ${ }^{*}$, NAN ZHOU and YANHUA QI \\ Department of Ophthalmology, The Second Affiliated Hospital of Harbin Medical University, \\ Harbin, Heilongjiang 150086, P.R. China
}

Received May 22, 2020; Accepted October 30, 2020

DOI: $10.3892 /$ etm.2020.9556

\begin{abstract}
The purpose of the present study was to assess the clinical characteristics of X-linked retinoschisis (XLRS) in a Chinese family over a 7-year period with the aim of identifying possible genetic mutations associated with this disease. A total of 2 male siblings from a family with XLRS were followed up for 7 years and the best-corrected visual acuity and data obtained using slit-lamp microscopy, indirect ophthalmoscopy, fundus photography, spectral domain-optical coherence tomography (OCT), fundus autofluorescence and fundus fluorescence (FFA) and multifocal electroretinograms (ERG) were examined. The coding regions of the retinoschisin 1 (RS1) gene were amplified by PCR and sequenced directly. The proband exhibited blurred vision at 12 years old and was indicated to exhibit a typical phenotype of XLRS at 30 years old. The elder brother exhibited blurred vision at 11 years old and was diagnosed with XLRS at 33 years old. There was no change in the best-corrected visual acuities in the two patients over the 7 years. The OCT results suggested that there were intraretinal cysts and macular atrophy in the eyes of the older sibling, whilst a 'spoke-wheel' pattern was present in the macula of the younger sibling. In addition, OCT examination revealed foveal schisis. FFA analysis indicated a hyperfluorescent signal in the central macula. Multifocal ERG recordings indicated that responses were markedly reduced in the central and outer rings bilaterally. The central retinal thickness of the younger sibling increased but the central retinal thickness of the older sibling was not changed during the 7 years. Sequencing analysis revealed that the mutation was c.366G>A (p.Trp122*) in exon 5 of Xp22.1. Gene mutation analysis indicated that the affected male siblings harbored a $\operatorname{Trp} 122^{*}$ (c.366G $>$ A) mutation, while the patients'
\end{abstract}

Correspondence to: Dr Yanhua Qi, Department of Ophthalmology, The Second Affiliated Hospital of Harbin Medical University, 246 Baojian Road, Harbin, Heilongjiang 150086, P.R. China E-mail: qyh86605643@126.com

${ }^{*}$ Contributed equally

Key words: retinoschisin 1 gene, gene mutation, congenital retinoschisis, macular schisis, optical coherence tomography mother was demonstrated to be a heterozygous carrier of the pathogenic mutation. To conclude, the present study discovered a novel XLRS mutation in a Chinese family, where the Trp122* mutation caused a significant change in the function of the RS1 protein. Over the 7 years of observation, although the vision was not significantly impaired in the two patients examined, the central retinal thickness of the younger sibling increased but the central retinal thickness of the older sibling was not altered.

\section{Introduction}

X-linked juvenile retinoschisis (XLRS) is a condition featuring the degeneration of the macula that commonly onsets early in males; major symptoms are vision loss and splitting or schisis of the retinal layer (1). Certain patients with this condition may also suffer from complications associated with vitreous hemorrhage and retinal detachment (1). XLRS is a monogenic, X chromosome-linked recessive disease that is caused by mutations in the retinoschisin (RS1) gene and affects between 1 in 5,000 and 1 in 25,000 males. By contrast, females who carry this trait do not suffer from loss of vision with the same frequency (2).

The RS1 gene is located at Xp22.1 and encodes that the 24-kDa discoidin domain-containing protein retinoschisin, which is secreted as a homo-oligomeric complex. RS1 is typically expressed in the retina and pineal gland (3), where it is predicted to serve as an adhesive protein in maintaining the structural and functional integrity of the retina (4).

Clinical retinoschisis is characterized by splitting that may occur in both the nerve fiber layer on the retinal surface and the deeper layers of the retina. Peripheral retinoschisis occurs in $<50 \%$ of affected individuals, whilst foveal involvement is present in all affected patients. Peripheral retinoschisis is commonly observed in the inferotemporal retina. With the use of optic coherence tomography (OCT), the diagnostic approach for XLRS has changed (5).

The purpose of the present study was to examine the clinical features of XLRS in a Chinese family over a 7-year monitoring period and to further identify the possible genetic mutations that are associated with this disease.

\section{Materials and methods}

Patients. A total of two patients from the same family were recruited at The Department of Ophthalmology, Second 
Table I. Clinical manifestations.

\begin{tabular}{lcccccc}
\hline Patient no. & Age (years) & Eye & 1st BCVA & 2nd BCVA & 3rd BCVA & Macular abnormalities \\
\hline III:3 (proband) & \multirow{2}{*}{30} & OD & $20 / 100$ & $20 / 100$ & $20 / 100$ & Schisis \\
& & OS & $20 / 80$ & $20 / 80$ & $20 / 100$ & Schisis \\
III:1 (elder brother) & \multirow{2}{*}{33} & OD & $20 / 50$ & N/A & $20 / 50$ & Schisis, atrophy \\
& & OS & $20 / 50$ & N/A & $20 / 50$ & Schisis, atrophy \\
\hline
\end{tabular}

BCVA, best-corrected visual acuity; OD, right eye; OS, left eye; N/A, not available; 1st, first visit; 2nd, follow-up 2 years later; 3rd, follow-up 7 years later.

Affiliated Hospital of Harbin Medical University (Harbin, China) between May and June 2011. They were healthy apart from having XLRS. The diagnosis of XLRS was made based on the presence of macular schisis with OCT examination. The present study was performed with approval from the Ethics Committee of The Second Affiliated Hospital of Harbin Medical University (Harbin, China). Informed consent was obtained from all participants.

Ophthalmic examinations. All patients were assessed for uncorrected visual acuity and best-corrected visual acuity, by fundus photography and spectral-domain OCT. The younger patient was examined by fundus autofluorescence and fundus fluorescence (FFA) and multifocal electroretinograms (ERG) during the first visit.

Mutation analysis. Blood samples were collected from all participants after obtaining informed consent according to the Declaration of Helsinki, which included the two patients, their parents and 100 healthy subjects (age range, 20-40 years; 50 males and 50 females) that were recruited at The Department of Ophthalmology, Second Affiliated Hospital of Harbin Medical University (Harbin, China) between May and December 2011, who were used as normal controls and were unrelated to the family. Individuals with ocular and systemic diseases were excluded. Genomic DNA was extracted from the peripheral blood using the Relaxgene Blood DNA System (Tiangen Biotech Co., Ltd.). RS1 gene coding regions and the flanking intron sequences were amplified by PCR (6) using a DNA polymerase from Takara Biotechnology Co., Ltd. The thermocycling conditions were as follows: Initial denaturation at $95^{\circ} \mathrm{C}$ for $10 \mathrm{~min}$, followed by 35 cycles of $95^{\circ} \mathrm{C}$ for $30 \mathrm{sec}, 55^{\circ} \mathrm{C}$ for $30 \mathrm{sec}$ and $72^{\circ} \mathrm{C}$ for $1 \mathrm{~min}$, and final extension at $72^{\circ} \mathrm{C}$ for $7 \mathrm{~min}$. The coding regions of the RS1 gene that encode retinoschisin were directly sequenced on an automated sequencer (ABI 3730xl Genetic Analyzer; Applied Biosystems; Thermo Fisher Scientific, Inc.) to perform mutation analysis. To identify the sequence variations, reference sequences of RS1 (NM_000330.3) were used.

\section{Results}

Clinical manifestations. A total of two male siblings were diagnosed with XLRS in the family (Fig. 1); they were both indicated to have macular abnormalities. The younger sibling was the proband, who presented with blurred vision at the age of 12 years, which could not be corrected. The patient was diagnosed at 30 years old with XLRS at The Second Affiliated Hospital of Harbin Medical University (Harbin, China) in May 2011 and was followed up for 7 years. At the first visit, a 'spoke wheel' pattern and macular retinoschisis were observed in the fundus (Fig. 2). Examination by OCT indicated macular schisis and there were increases in the thickness of the macula in both eyes compared with that in normal eyes. The older male sibling of the proband visited 1 month later, he started exhibiting blurred vision at 11 years old, was diagnosed at 33 years old, and bilateral macular schisis, intraretinal cysts and macular atrophy were observed in this patient (Table 1). Over 7 years, notable increases in the thickness of the macula were detected in both eyes of the proband, whilst macular atrophy persisted in the older sibling. There were no changes in visual acuity in both patients (Figs. 3 and 4).

Fundus fluorescence angiography. FFA images acquired at the first visit revealed a 'spoke wheel' pattern of hyperfluorescence in the central macular area of the proband (Fig. 5).

ERG. Multifocal ERG (mfERG) was recorded from the central $30^{\circ}$ of the visual field of the proband, where responses were revealed to be reduced bilaterally in the central and outer rings at the first visit. The mfERG results suggested central cone dysfunction affecting not only the macular area but across the central $30^{\circ}$ of the visual field tested in each eye (Fig. 6).

Genetic analysis. The two patients were revealed to carry a genetic mutation in the RS1 gene; the mutation was determined to be localized in exon 5 (c.366G $>$ A). This $\mathrm{G}>\mathrm{A}$ substitution changed the amino acid from tryptophan to a stop codon, which produced a nonfunctional and truncated protein that was 22 amino acids in length.

None of the healthy family members or the 100 control subjects examined tested positive for this mutation (Fig. 7). The mother of the proband was demonstrated to be a heterozygous carrier of the mutation without the manifestation of any symptoms. Base changes were neither identified in the sequences of other normal members of the family nor in the normal control group. The 3D protein structures of RS1 were analyzed using the Phyre 2 software. Molecular modeling indicated that the p.W122* substitution significantly changed the secondary structure of the RS1 protein and made the secondary structure shorter, which was caused by the mutation of c.366G $>$ A to 


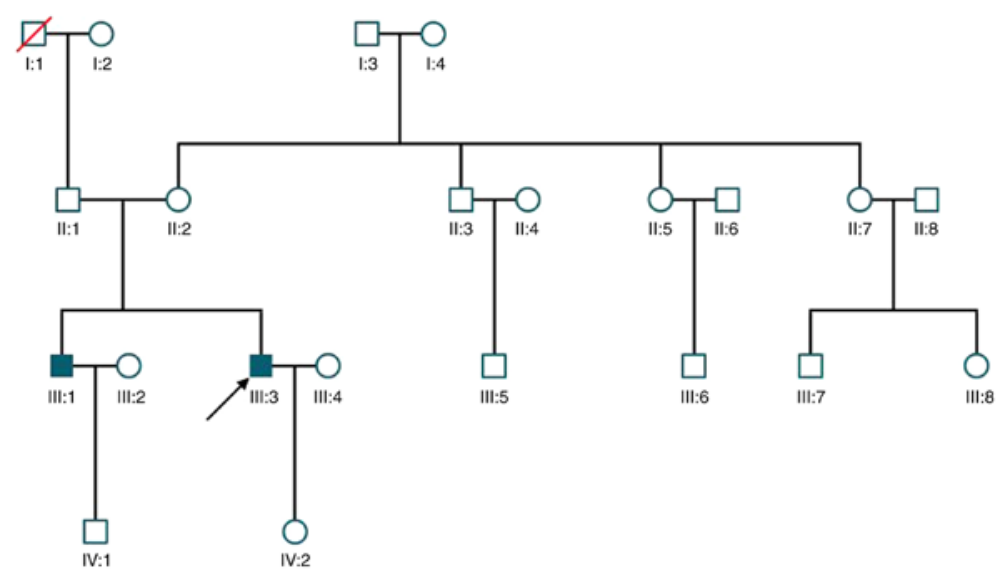

Figure 1. Pedigree chart of the patients. The black arrow indicates the proband.
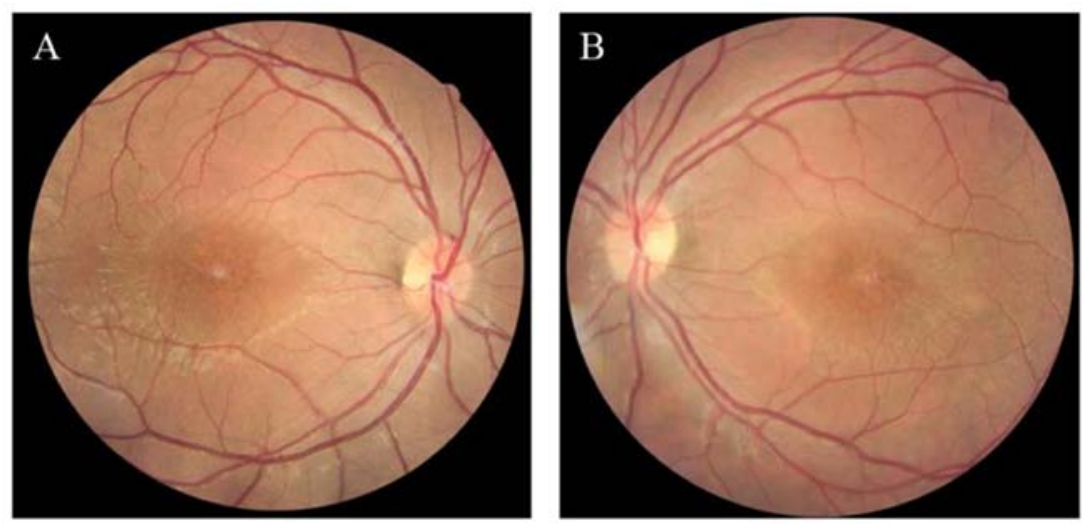

Figure 2. Fundus images of the younger sibling (the proband) at the first visit. (A) Right eye; (B) left eye. A typical 'spoke wheel'-like appearance in the central macula of both eyes was observed.
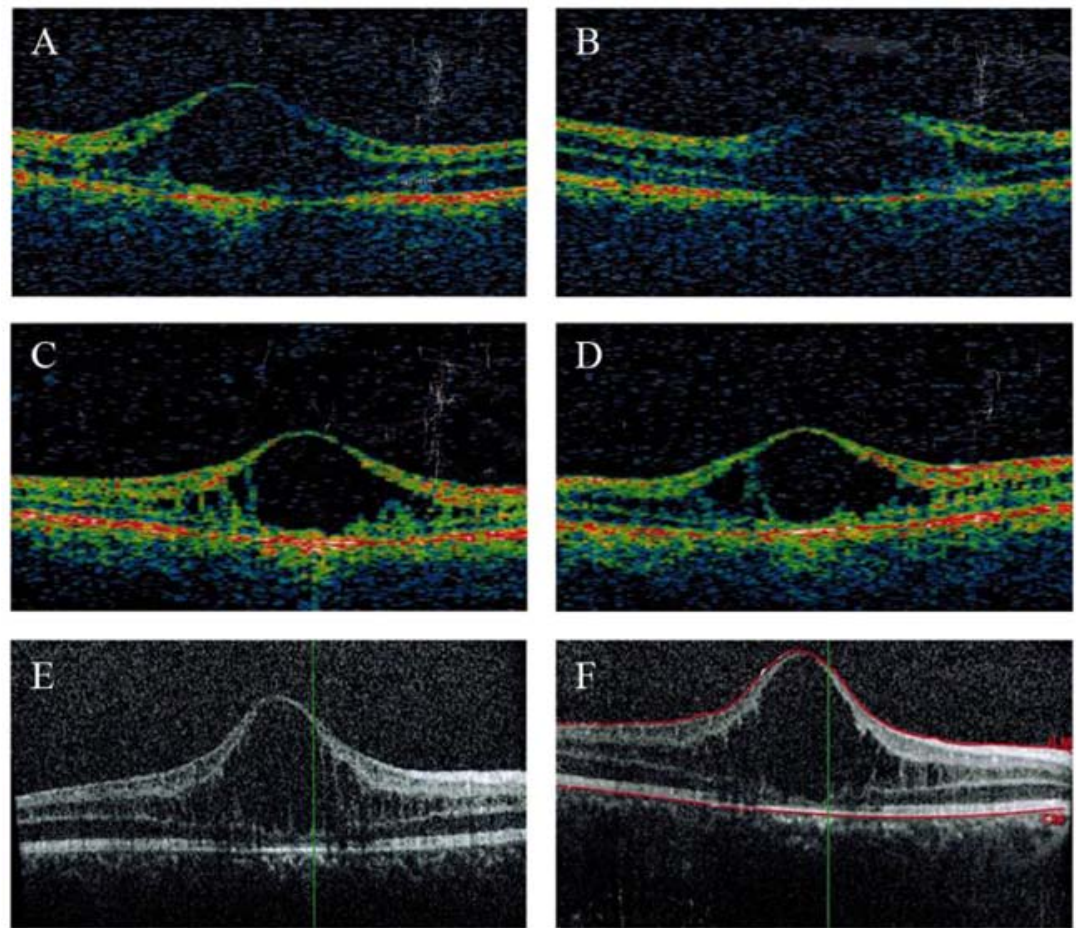

Figure 3. Spectral domain-optical coherence tomography images of the younger sibling (the proband). (A) Right eye and (B) left eye indicating foveal schisis at the first visit. (C) Right eye and (D) left eye indicating foveal schisis 2 years later. (E) Right eye and (F) left eye indicating foveal schisis 7 years later. Increased thickness was observed in the fovea over 7 years. 

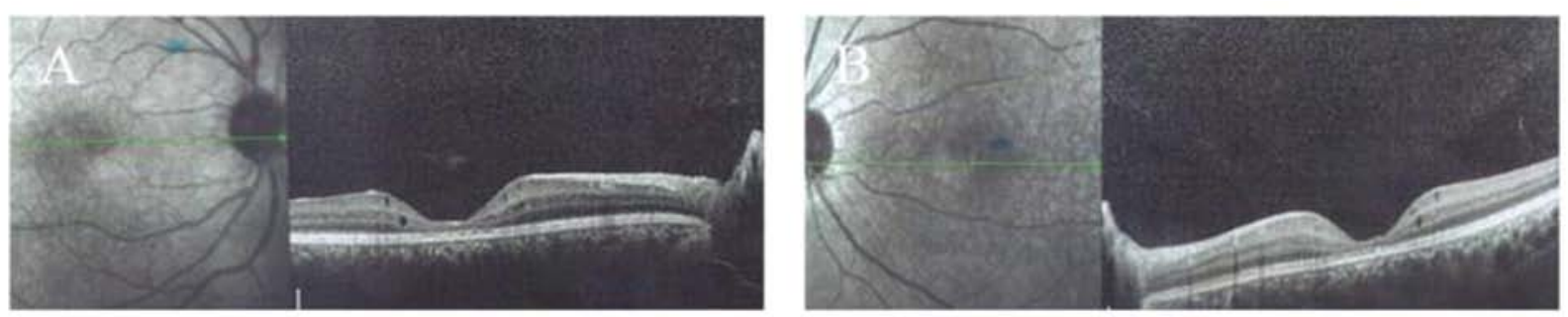

Figure 4. Spectral domain-optical coherence tomography images of the older male sibling of the proband. (A) Right eye; (B) left eye. Foveal schisis and macular atrophy were observed.
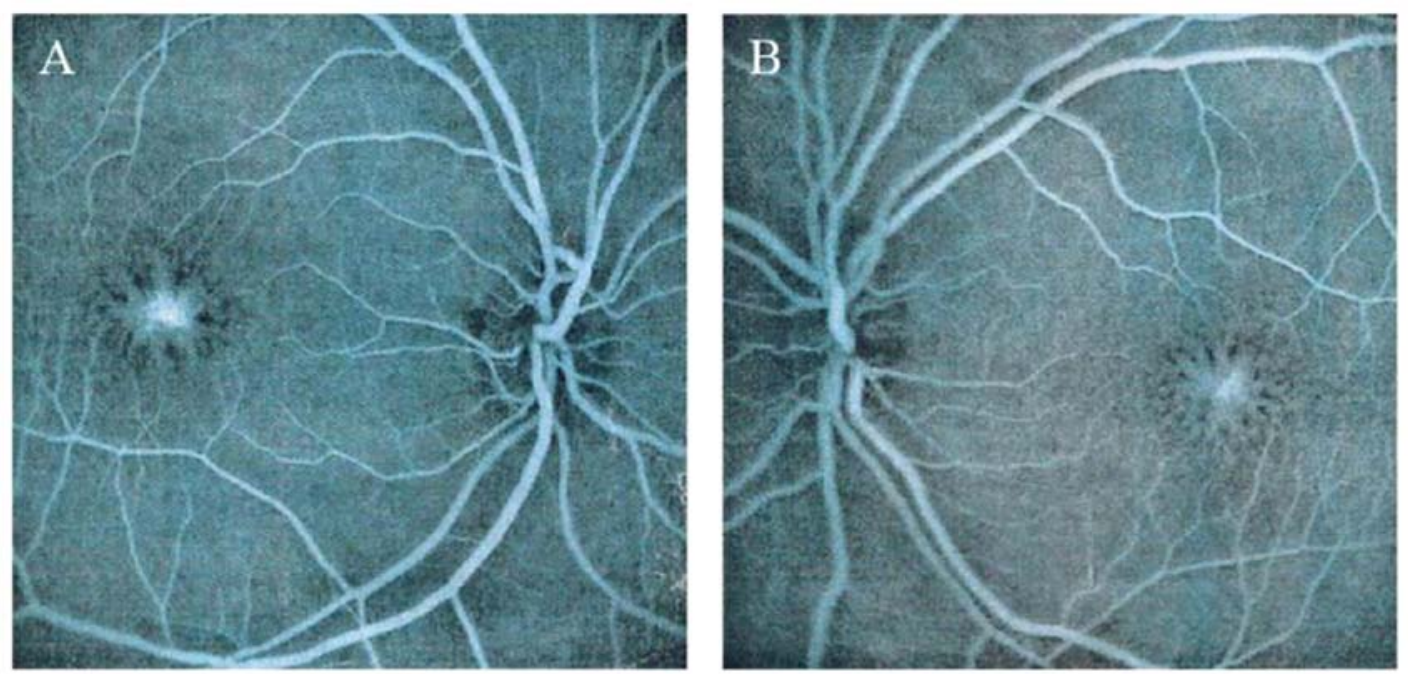

Figure 5. FFA analysis of the younger sibling (the proband). (A) Right eye; (B) left eye. The younger patient had a 'spoke-wheel' pattern of hyper FFA in the macular area. FFA, fundus fluorescence angiography.

$$
\text { Amplitudes P1(b) }
$$

$$
\begin{array}{r}
I 500 \mathrm{nV} \\
50 \mathrm{~ms} \\
\text { Amp.P1 }\left[\mathrm{nV} / \mathrm{deg}^{2}\right]
\end{array}
$$

3D Amplitudes P1(b)

\begin{tabular}{rrrrr}
$20^{\circ}$ & $10^{\circ}$ & $0^{\circ}$ & $10^{\circ}$ & $20^{\circ}$ \\
\hline
\end{tabular}

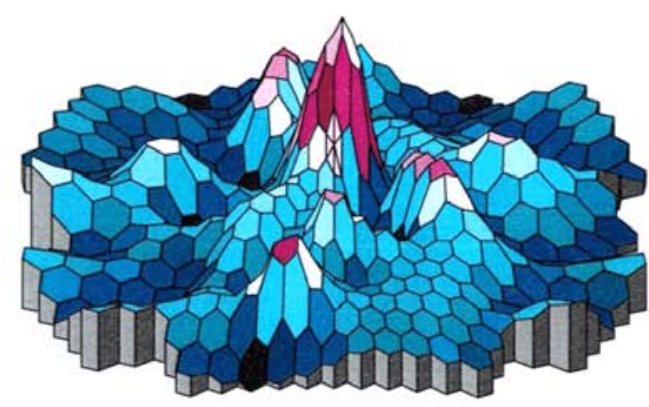

Figure 6. Electroretinogram analysis of the younger sibling (left eye of the proband). Responses were reduced bilaterally in the central and outer rings (central $30^{\circ}$ of the visual field).

generate a stop codon. This observation suggested that this mutation is likely to markedly impact the protein function (Fig. 8).

There are currently no effective treatments for XLRS. The two patients did not receive any treatment but were only clinically followed. It has been reported that carbonic anhydrase inhibitors (CAIs), which belong to sulfanilamide drugs, were effective in improving cystoid macular edema in patients with XLRS (7). The elder brother's macula was already atrophic at presentation, and although the younger brother's macular thickness increased, he had a history of sulfanilamide allergy, therefore he was not treated with CAIs. 
A

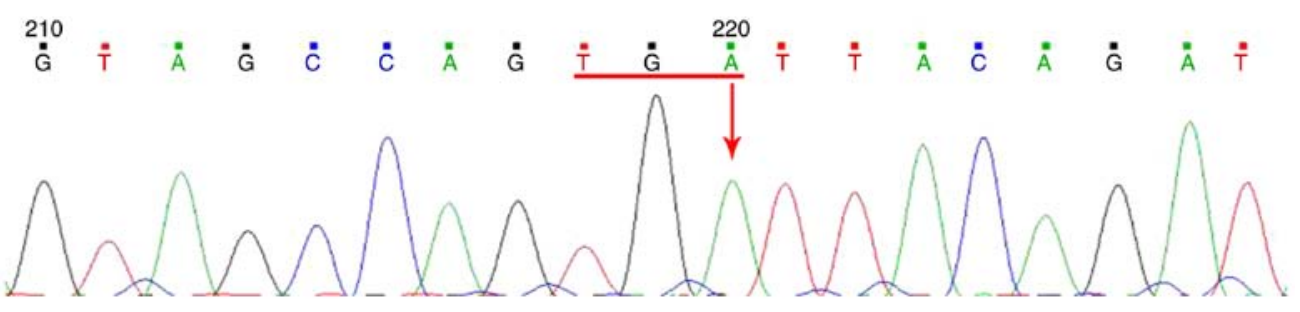

B

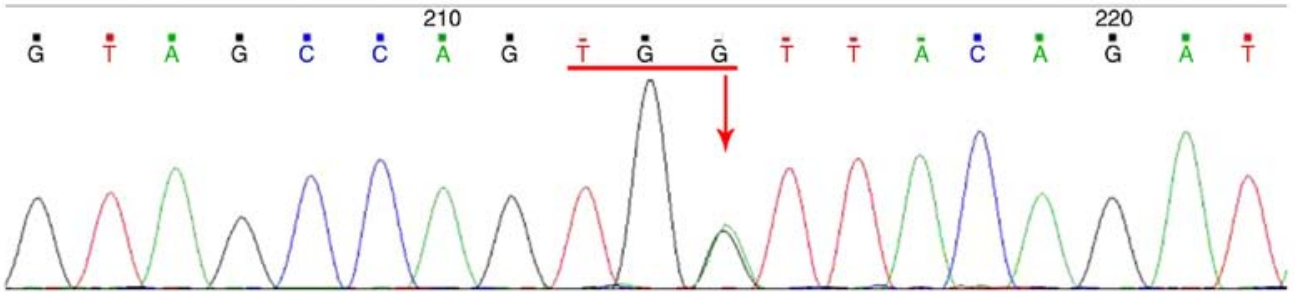

C

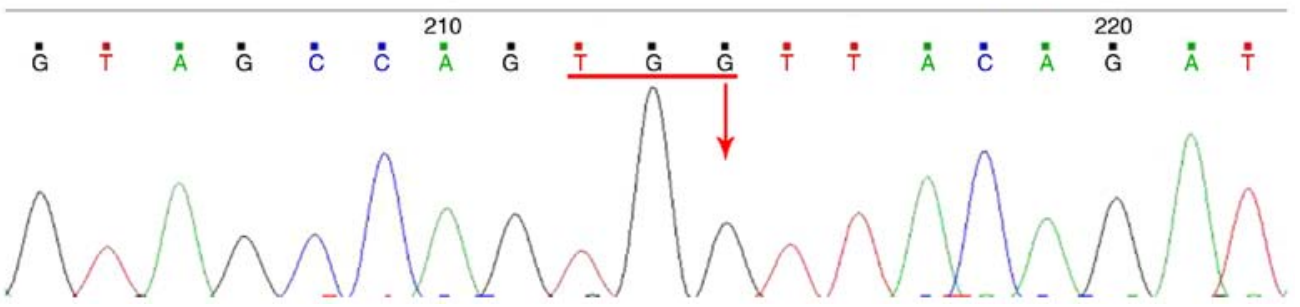

Figure 7. Partial sequence of the RS1 gene at exon 5. Sequencing patterns indicated that the heterozygous c.366G>A missense mutation was present in all affected subjects, but not in the control subjects. (A) Arrow indicates the location of the younger brother's base mutation. (B) The mother of the proband was a heterozygous carrier of the mutation without clinical symptoms. (C) No base changes were identified in the sequences of unaffected members of the family or the normal control group. RS1, retinoschisin 1.
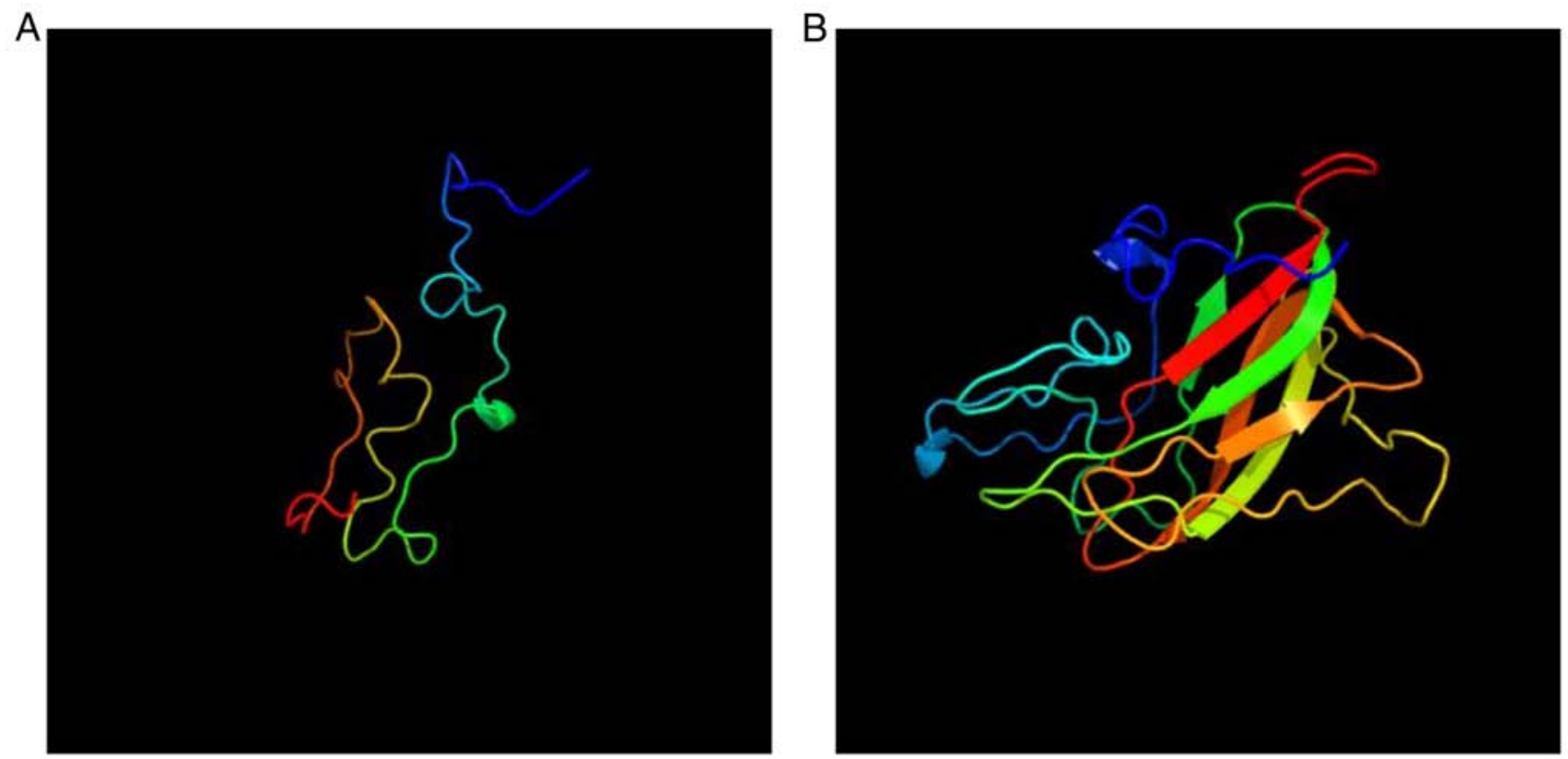

Figure 8. Predicted secondary structure of the mutant RS1 protein. (A) Mutant and (B) wild-type 3D protein structures of RS1 obtained using the Phyre2 software. Molecular modeling indicated that the p.W122* substitution significantly shortened the secondary structure of the RS1 protein, which was caused by the c.366G $>$ A mutation to generate a stop codon. RS1, retinoschisin 1 .

\section{Discussion}

XLRS is a congenital disease that was first described by Haas (8) in 1898. It causes changes to the retinal structure early in life. A number of studies have previously demonstrated that XLRS is a neurodevelopmental abnormality caused by gene mutations, leading to retinoschisis. In total, $\sim 251$ different mutations in the RS1 gene have been reported to cause XLRS, 
the clinical characteristics of which include splitting of the retina and visual impairment. Kjellström et al (9) previously followed up 10 patients for 12 years and determined no changes in visual acuity after childhood. Similar results were also reported by another study (10), suggesting XLRS to be a progressive retinal degenerative condition with a slow onset. Although a 'spoke wheel'-like appearance in the macula is the most typical characteristic feature of XLRS, subretinal fibrosis, macular atrophy, mimicking maculopathy, vitreous hemorrhage, peripheral retinoschisis and peripheral pigmentary changes are also occasionally present in patients with XLRS (11).

XLRS is a clinically heterogeneous disease with $>100$ different mutations in the RS1 gene now known to cause this disease, where two patients in a family with the same mutation may present with different phenotypes. In a previous study, Kondo et al (12) reported that foveal schisis was more frequently associated with missense mutations in 67 Japanese patients from 56 families affected with XLRS, where peripheral schisis was detected in 50 and $67 \%$ of the eyes of patients harboring truncation and missense mutations, respectively.

$\mathrm{Hu}$ et al (13) examined 30 patients suspected to exhibit XLRS and 51 patients with confirmed XLRS in China and identified 28 mutations associated with this disease, 8 of which were novel. Tian et al (14) reported on 6 families with XLRS in China, whilst certain sporadic cases of XLRS and novel mutations were also reported in China (15).

The present study reported on the clinical characteristics and data obtained by OCT, FFA, ERG and RS1 gene mutation analysis of 2 patients from a Chinese family who were followed up for 7 years. The eyes of the younger sibling had a 'spoke wheel'-like appearance, whereas those of the older sibling exhibited intraretinal cysts and macular atrophy. The younger sibling was followed up for 7 years, who was suffering from blurred vision at 12 years of age but no change in visual acuity was observed over the 7 years after presentation in the hospital. OCT analysis revealed a minor change in the central macula; however, the macular thickness was determined to be increased during 7 years of follow-up. The older sibling was diagnosed with XLRS at the age of 33 years and atrophy persisted in the central macula after 7 years.

In addition, the present study also identified a novel genetic mutation in the RS1 gene of the two patients examined, which was localized in exon 5 (c.366 G>A), where this G>A substitution changed the amino acid from tryptophan to a stop codon, producing a truncated nonfunctional 22-amino acid protein. Subsequent molecular modeling indicated that p.W122* substitution significantly shortened the secondary structure of the $\mathrm{RS} 1$ protein. This mutation was indicated to be clustered in the discoidin domain and occurred within the conserved residues, where several studies have previously reported that mutations may cause protein misfolding and retention in the endothelial reticulum (16). This observation suggested that this mutation may impact RS1 function.

In 1997, Sauer et al (17) discovered that the causative gene of XLRS was RS1 located at Xp22. RS1 is expressed in photoreceptor cells and bipolar cells, it contains 6 exons and encodes a secreted protein called the retinal splitting protein (18). The retinal splitting protein is comprised of 224 amino acids and contains a highly hydrophobic signal peptide. This signal peptide is cleaved by peptidases to form a mature protein serving as a connection between cells in the inner nuclear layer, which is closely associated with synaptic connections of photoreceptors, where the bipolar cells are located (19). The retinal splitting protein contains a disc-like domain that consists of 157 amino acids and 10 cysteines, which is encoded by exons 4, 5 and 6 . This domain is highly conserved and has been previously associated with cell adhesion and signal transmission throughout evolution (20). The discoid domain receptor is a transmembrane receptor that is able to interact with collagen, mediate adhesion between cells and regulate the extracellular matrix (21).

RS1 is mainly expressed in photoreceptor and bipolar cells, potentially providing a reason for the lack of systemic tissue abnormalities in male patients (22). Examination of female carriers may prove difficult due to the absence of clinical symptoms (22). However, a previous study has suggested that female carriers may also exhibit an abnormal ERG performance, with observed splitting in the periphery or center of the retina (22). The division of the retina may be due to the inactivation of the $\mathrm{X}$ chromosome, resulting in this abnormal clinical manifestation (22).

It has been indicated that mutations in the RS1 gene cause dysfunction in the secretion of protein products and loss of adhesion function, impaired intercellular communication, weakened adhesion between the retinal layers and the formation of internal retinal fission cavities (23). The new mutation identified in the present cases whose mother was determined to be a carrier leads to protein truncation. By applying a software prediction of the changes in protein structure, it was indicated that the structure of the protein was significantly changed due to the mutation. The site was indicated to be located in the highly conserved disc-like domain of the RS1 gene (24). It may be speculated that this mutation affects the communication between cells and adhesion between the retinal layers, resulting in the splitting of the inner retina (25).

The biochemical mechanisms of XLRS remain poorly understood. There are currently no effective treatments for XLRS. Congenital retinal splitting has a slow onset and is difficult to observe during stable phases of the disease. When complications occur, they may be treated symptomatically (26). A number of studies on patients with XLRS indicated that the vision of adult patients deteriorates, whilst the vision of the majority of patients aged $>70$ years falls $<0.1$ (27). It is promising that the retinal structure and function improved after gene transfer therapy in a mouse RS1 knockout model of XLRS $(28,29)$. Bashar et al $(30)$ previously reported that extracellular delivery of RS1 rescued the structural and functional deficits in the RS1h knockout mouse model, where this ex vivo gene therapy approach was able to inhibit disease progression. Zeng et al (28) injected RS1h complementary DNA into the eyeballs of adult RS1h knockout mice, which reversed the abnormal negative waveform of ERG, restored the positive $b$ wave and led to the expression of the retinal splitting protein in the entire layer of the retina. Therefore, it is believed that gene transfer may be an effective treatment strategy for XLRS, which brings optimism for future interventions of this disease. 
Zhao et al (26) previously studied 32 eyes with severe complications that underwent vitrectomy and determined that vitreoretinal surgery significantly improved visual acuity and restored the anatomic structure of the retina. In addition, carbonic anhydrase inhibitors may be helpful in reducing the cavities and retinal thickness (31). Although significant progress has been made in recent years, numerous questions, for example the potential effects of gene therapy, should be further explored.

Since there is as yet no effective treatment for XLRS, screening for gene mutations is vital for understanding the pathogenesis of XLRS to explore novel effective treatment methods for this disease.

\section{Acknowledgements}

Not applicable.

\section{Funding}

No funding was received.

\section{Availability of data and materials}

All data generated or analyzed during this study are included in this published article.

\section{Authors' contributions}

YQ designed the study; NZhang and YP performed the experiments; NZhou acquired clinical data; NZhang wrote and revised the manuscript. All authors read and approved the final manuscript.

\section{Ethics approval and consent to participate}

The present study was performed with approval from the Ethics Committee of The Second Affiliated Hospital of Harbin Medical University (Harbin, China). Informed consent was obtained from all participants.

\section{Patient consent for publication}

The proband and the elder brother provided consent for publication.

\section{Competing interests}

The authors declare that they have no competing interests.

\section{References}

1. George ND, Yates JR and Moore AT: X linked retinoschisis. Br J Ophthalmol 79: 697-702, 1995

2. Wieacker P, Wienker J, Dallapiccola B, Bender K, Davies KE and Ropers $\mathrm{HH}$ : Linkage relationships between retionschisis, $\mathrm{Xg}$, and a cloned DNA seqence from the distal short arm of the X chromosome. Hum Genet 64: 143-145, 1983.

3. Takada Y, Fariss RN, Tanikawa A, Zeng Y, Carper D, Bush R and Sieving PA: A retinal neuronal developmental wave of retinoschisin expression begins in ganglion cells during layer formation. Invest Ophthalmol Vis Sci 45: 3302-3312, 2004.
4. Wu WW, Wong JP, Kast J and Molday RS: RS1, a discoidin domain-containing retinal cell adhesion protein associated with $\mathrm{X}$-linked retinoschisis, exists as a novel disulfide-linked octamer. J Biol Chem 280: 10721-10730, 2005.

5. Renner AB, Kellner U, Fiebig B, Cropp E, Foerster MH and Weber BHF: ERG variability in X-linked congenital retinoschisis patients with mutation in the RS1 gene and the diagnostic importance of fundus autofluorescence and OCT. Doc Ophthalmol 116: 97-109, 2008

6. Wang NK, Liu LL, Chen HM, Tsai S, Chang TC, Tsai TH, Yang CM, Chao AN, Chen KJ, Kao LY, et al: Clinical presentations of X-linked retinoschisis in Taiwanese patients confirmed with genetic sequencing. Mol Vis 21: 487-501, 2015.

7. Thobani A and Fishman GA: The use of carbonic anhydease inhibitors in the retreatment of cystic macular lesions in retinitis pigmentosa and X-linked retinoschisis. Retina 31: 312-315, 2011.

8. Haas J: Über das zusammenvorkommen von veraenderungen der retina und choroides. Arch Augenheilkd 37: 343-348, 1898.

9. Kjellström S, Vijayasarathy C, Ponjavic V, Sieving PA and Andréasson S: Long-term 12 year follow-up of X-linked congenital retinoschisis. Ophthalmic Genet 31: 114-125, 2010.

10. Kellner U, Brummer S, Foerster MH and Wessing A: X-linked congenital retinoschisis. Graefes Arch Clin Exp Ophthalmol 228: 432-437, 1990.

11. Apushkin MA, Fishman GA and Janowica MJ: Correlated of optical coherence tomography findings with visual acuity and macular lesions in patients with $\mathrm{X}$-linked retinoschisis. Ophthalmology 112: 495-501, 2005.

12. Kondo H, Oku K, Katagiri S, Hayashi T, Nakano T, Iwata A, Kuniyoshi K, Kusaka S, Hiyoshi A, Uchio E, et al: Novel mutations in the RS1 gene in Japanese patients with X-linked congenital retinoschisis. Hum Genome Var 6: 3, 2019.

13. Hu QR, Huang LZ, Chen XL, Xia HK, Li TQ and Li XX: Genetic analysis and clinical features of $\mathrm{X}$-linked retinoschisis in Chinese patients. Sci Rep 7: 44060, 2017.

14. Tian R, Jiang RX and Chen YX: Genetic and phenotypic characteristics of six Chinese families with X-linked juvenile retinoschisis. Chin Med J (Engl) 126: 4392-4394, 2013.

15. Huang XF, Tu CS, Xing DJ, Gan DK, Xu GZ and Jin ZB: R102W mutation in the RS1 gene responsible for retinoschisis and recurrent glaucoma. Int J Ophthalmol 7: 169-172, 2014.

16. Wu WW and Molday RS: Defective discoidin domain structure subunit assembly, and endoplasmic reticulum processing of retinoschisin are primary mechanisms responsible for X-linked retinoschisis. J Biol Chem 278: 28139-28146, 2003.

17. Sauer CG, Gehrig A, Warneke-Wittstock R, Marquardt A Ewing CC, Gibson A, Lorenz B, Jurklies B and Weber BH: Positional cloning of the gene associated with X-linked juvenile retinoschisis. Nat Genet 17: 164-170, 1997.

18. Gehrig AE, Warneke-Witstock R, Sauer CG and Weber BH: Isolation and characterization of the murine $\mathrm{X}$-linked juvenile retinoschisis (Rs 1 h) gene. Mamm Genome 10: 303-307, 1999.

19. Robert SM, Ulrich K and Bernhard HF: X-linked juvenile retinoschisis: Clinical diagnosis, genetic analysis, and molecular mechanisms. Prog Retin Eye Res 31: 195-212, 2012.

20. Baumgartner S, Hofmann K, Chiquet-Ehrismann R and Bucher P: The discoidin domain family revisited: New members from prokaryotes and a homology-based fold prediction. Protein Sci 7: 1626-1631, 1998.

21. Raymond A, Ensslin MA and Shur BD: SED1/MFG-E8: A bi-motif protein that orchestrated diverse cellular interaction. J Cell Biochem 106: 957-966, 2009.

22. Kim LS, Seiple W, Fishman GA and Szlyk JP: Multifocal ERG findings in carriers of $\mathrm{X}$-linked retinoschisis. Doc Ophthalmol 114: 21-26, 2007.

23. Grayson C, Reid SN, Ellis JA, Rutherford A, Sowden JC, Yates JR, Farber DB and Trump D: Retinoschisin, the X-linked retinoschisis protein, is a secreted photoreceptor protein, and is expressed and released by Weri-Rb1 cell. Hum Mol Genet 9: 1873-1879, 2000.

24. Fraternali F, Cavallo L and Musco G: Effect of pathological mutations on the stability of a conserved amino acid triad in retinoschisin. FEBS Lett 544: 21-26, 2003.

25. Sergeev YV, Caruso RC, Meltzer MR, Smaoui N, MacDonald IM and Sieving PA: Molecular modeling of retinoschisin with functional analysis of pathogenic mutations from human X-linked retinoschisis. Hum Mol Genet 19: 1302-1313, 2010. 
26. Zhao C, Zhang Q, Jin HY and Zhao PQ: Clinical observations of vitreoretinal surgery for four different phenotypes of X-linked congenital retinoschisis. Int J Ophthalmol 11: 986-990, 2018

27. Tantri A, Vrabec TR, Cu-Unjieng G, Frost A, Annesley WH Jr and Donoso LA: X-linked retinoshisis: A clinical and molecular genetic review. Surv Ophthalmol 49: 214-230, 2004.

28. Zeng Y, Takada Y, Kjellstrom S, Hiriyanna K, Tanikawa A, Wawrousedk E, Smaoui N, Caruso R, Bushu RA and Sieving PA: RS-1 gene delivery to an adult Rs1h knockout mouse model restores ERG b-wave with reversal of the electronegative waveform of X-linked retinoschisis. Invest Ophthalmol Vis Sci 45: 3279-3285, 2004.

29. Min SH, Molday LL, Seeliger MW, Dinculescu A, Timmers AM, Janssen A, Tonagel F, Tanimoto N, Weber BH, Molday RS and Hauswirth WW: Prolonged recovery of retinal stucture/function after gene therapy in an Rs1h-deficient mouse model of X-linked juvenile retinoschisis. Mol Ther 12: 644-651, 2005.
30. Bashar AE, Metcalfe AL, Viringipurampeer IA, Yanai A, Gregory-Evans CY and Gregory-Evans K: An ex vivo gene therapy approach in X-linked retinoschisis. Mol Vis 22: 718-733, 2016.

31. Apushkin MA and Fishman GA: Use of dorzolamide for patients with X-linked retinoschisis. Retina 26: 741-745, 2006.

(i) $\ominus$ This work is licensed under a Creative Commons Attribution-NonCommercial-NoDerivatives 4.0 International (CC BY-NC-ND 4.0) License. 